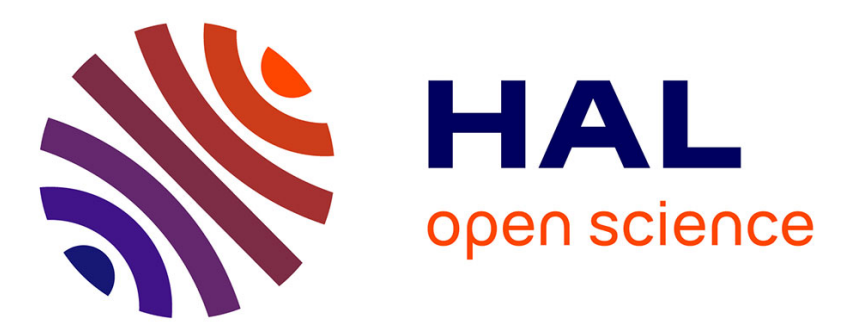

\title{
A transverse isotropic equivalent fluid model combining both limp and rigid frame behaviors for fibrous materials
}

Benoit Nennig, Rémy Binois, Nicolas Dauchez, Emmanuel Perrey-Debain, Félix Foucart

\section{- To cite this version:}

Benoit Nennig, Rémy Binois, Nicolas Dauchez, Emmanuel Perrey-Debain, Félix Foucart. A transverse isotropic equivalent fluid model combining both limp and rigid frame behaviors for fibrous materials. Journal of the Acoustical Society of America, 2018, 143 (4), pp.2089 - 2098. 10.1121/1.5030925 . hal-01768467

\section{HAL Id: hal-01768467 \\ https://hal.science/hal-01768467}

Submitted on 17 Apr 2018

HAL is a multi-disciplinary open access archive for the deposit and dissemination of scientific research documents, whether they are published or not. The documents may come from teaching and research institutions in France or abroad, or from public or private research centers.
L'archive ouverte pluridisciplinaire HAL, est destinée au dépôt et à la diffusion de documents scientifiques de niveau recherche, publiés ou non, émanant des établissements d'enseignement et de recherche français ou étrangers, des laboratoires publics ou privés. 


\title{
A transverse isotropic equivalent fluid model combining both limp and rigid frame behaviors for fibrous materials
}

\author{
Benoit Nenniga) and Rémy Binois \\ Institut supérieur de mécanique de Paris (SUPMECA), Laboratoire Quartz EA 7393, \\ 3 rue Fernand Hainaut, 93407 Saint-Ouen, France. \\ Nicolas Dauchez, Emmanuel Perrey-Debain, and Félix Foucart \\ Sorbonne universités, Université de Technologie de Compiègne, \\ Laboratoire Roberval, UMR CNRS 7337, CS 60319, 60203 Compiègne cedex, France.
}

(Dated: Feb. 2018)

\begin{abstract}
Due to the manufacturing process, some fibrous materials like glasswool may be transversely isotropic (TI): fibers are mostly parallel to a plane of isotropy within which material properties are identical in all directions whereas properties are different along the transverse direction. The behavior of TI fibrous material is well described by the TI Biot's model, but it requires to measure several mechanical parameters and to solve the TI Biot's equations.

This paper presents an equivalent fluid model that can be suitable for TI materials under certain assumptions. It takes the form of a classical wave equation for the pressure involving an effective density tensor combining both limp and rigid frame behaviors of the material. This scalar wave equation is easily amenable to analytical and numerical treatments with finite element method.

Numerical results, based on the proposed model, are compared with experimental results obtained for two configurations with a fibrous material. The first concerns the absorption of an incident plane wave impinging on a fibrous slab and the second corresponds to the transmission loss of a splitter-type silencer in a duct. Both configurations highlight the effect of the sample orientation and give an illustration of the unusual TI behavior for fluids.
\end{abstract}

This archived file is not the final published version of "B. Nennig, R. Binois, N. Dauchez, E. PerreyDebain, and F. Foucart. A transverse isotropic equivalent fluid model combining both limp and rigid frame behaviors for fibrous materials. J. Acoust. Soc. Am., 143(4):2089-2098, 2018.". Contact the publisher for reprint or permission to use the material in any form. The definitive publisherauthenticated version is available online at https://doi. org/10.1121/1.5030925.

PACS numbers: 43.20.Gp, 43.20.Mv, 43.50.Gf

Keywords: Transverse isotropic fluid, metafluids, Porous materials, Silencers, waveguides

\section{INTRODUCTION}

Porous materials, such as those used for sound absorption, are made of a fluid phase and a solid phase. Their dynamic behavior is well described by the Biot theory, which takes into account the skeleton motion coupled with the fluid embedded inside the pore network ${ }^{1,2}$. In cases of a large contrast between the fluid and the solid stiffness, simplified modeling approach can be used. This yields classically i) the rigid frame model ${ }^{2}$ for which the solid phase is considered as motionless and ii) the limp model ${ }^{3-6}$ where skeleton stiffness is very small in comparison with the saturating fluid bulk modulus. In this case, only inertial effects due to the solid frame motion are retained.

Though originally developed for isotropic materials, the Biot theory has been extended to anisotropic materials. However, the mechanical and acoustical characterization of such materials remains challenging and recent works have been dedicated to the measurement of elas-

a) Electronic address: benoit.nennig@supmeca.fr; Corresponding author. tic and acoustical parameters ${ }^{7-12}$ either by direct or by inverse methods. Acoustic parameters can also be estimated via a micro-macro approach when a representative elementary volume is known by imaging technique ${ }^{13-15}$. From a computational point of view, Biot's equations can be solved by the finite element method ${ }^{16,17}$ (see also the references therein). In some situations, semi-analytical techniques such as the Transfer Matrix Method ${ }^{18}$ and the plane wave decomposition ${ }^{19}$ can also be used. In these papers, it is illustrated how the anisotropic nature of the material can have a strong impact on sound absorbing properties.

Due to manufacturing process, some fibrous materials like wools may be transversely isotropic: wool fibers are mostly ${ }^{14}$ parallel to a plane of isotropy $(I)$ within which material properties are the same in all directions whereas properties are different along the transverse direction $(T)$, which is normal to this plane (see Fig. 1). These TI materials have been studied for many years ${ }^{20-23}$ (a quick survey can be found in $^{2}$ (section 3.7, p. 39)).

The mathematical description of wave propagation in such materials is usually made using the classical rigid frame assumption. This permits to derive a simplified equivalent fluid model involving a scalar bulk modulus and effective densities which take the form 
of a tensor 24,25 . This class of fluid is also present in superfluid ${ }^{26,27}$, in the low frequency limit (homogenization) for multilayered materials ${ }^{24}$, in sonic crystals or metamaterials $^{28-30}$.

The aim of this paper is to extend the equivalent fluid model approach to TI materials, such as soft fibrous, for which the rigid frame assumption remains valid only in the plane of isotropy (stiff directions) while inertial effects due to the frame motion along the transverse direction are retained. The interest is twofold: (i) it permits the analysis of the leading sound absorbing properties of this class of materials without the knowledge of elastic parameters which are known to be difficult to measure and (ii) the equivalent fluid model takes the form of a scalar wave equation which is easily amenable to analytical and numerical treatments with finite element method (FEM).

The paper is organized as follows: first, the full Biot's equations related to TI poroelastic materials are recalled and the equivalent TI fluid model is derived. Two classes of analytical solutions are investigated: plane wave solutions and guided modes. In order to deal with more general configurations, the numerical treatment of the wave equation using FEM is also presented. In a second part of the paper, some experimental results are shown. Two specific scenarios are investigated: one concerns the sound absorption properties at normal incidence, the other one is related to the transmission loss for dissipative silencers whose dimensions are very similar to recent applications already published by the present authors ${ }^{31,32}$. Results are discussed and compared with numerical simulations.

\section{THEORETICAL MODEL}

\section{A. Wave equation for the pressure}

Original Biot's equations ${ }^{1,33}$ are written in terms of solid and fluid displacements $\mathbf{u}$ and $\mathbf{U}$ or with their combination. In the present discussion, it is more convenient for the analysis to use an alternative formulation based on the in vacuo solid stress tensor $\hat{\boldsymbol{\sigma}}$ and proposed by Dazel et $a l .{ }^{5}$. The formulation involves the skeleton displacement $\mathbf{u}$ and the quantity $\mathbf{u}^{w}$ which, in the present situation, is equal to the total displacement defined as $\mathbf{u}^{t}=\phi \mathbf{U}+(1-\phi) \mathbf{u}$ (here, $\phi$ is the porosity). This formulation has been extended to TI poroelastic materials ${ }^{18,34}$. We recall here the TI governing equations from Khurana et $a l .{ }^{18}$

$$
\begin{array}{r}
\nabla \cdot \hat{\boldsymbol{\sigma}}(\mathbf{u})=-\omega^{2} \boldsymbol{\rho}_{s} \mathbf{u}-\omega^{2} \boldsymbol{\gamma} \boldsymbol{\rho}_{\mathrm{eq}} \mathbf{u}^{w} \\
K_{\mathrm{eq}} \nabla\left(\nabla \cdot \mathbf{u}^{w}\right)=-\omega^{2} \gamma \boldsymbol{\rho}_{\mathrm{eq}} \mathbf{u}-\omega^{2} \boldsymbol{\rho}_{\mathrm{eq}} \mathbf{u}^{w}
\end{array}
$$

where, $K_{\text {eq }}$ is the bulk modulus of the fluid phase (see appendix $\mathrm{A}$ ).

The coupling coefficient $\gamma$ and the effective density $\boldsymbol{\rho}_{\mathrm{eq}}$

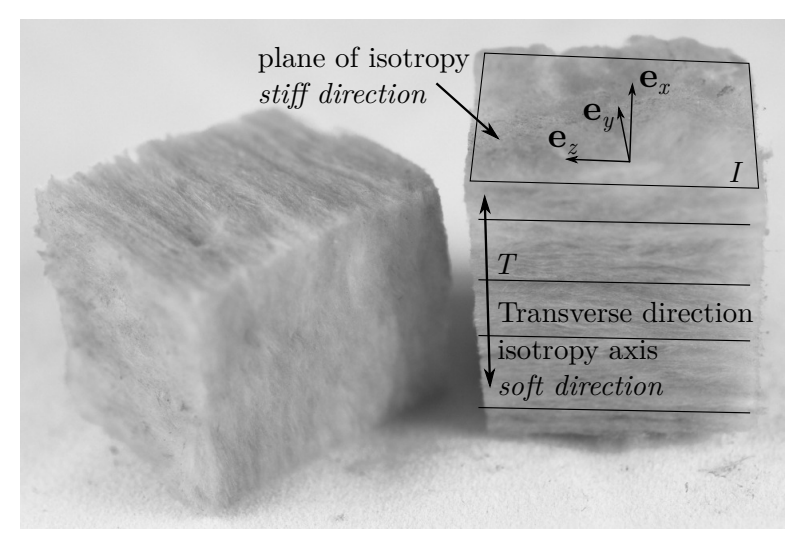

FIG. 1. Transverse isotropic material and associated coordinate system, when the sample is aligned on its principal axis.

and $\boldsymbol{\rho}_{s}$ can be linked to the Biot effective densities

$$
\begin{aligned}
\gamma & =\phi\left(\boldsymbol{\rho}_{22}^{-1} \boldsymbol{\rho}_{12}-\frac{Q}{R} \mathbf{I}\right), \\
\boldsymbol{\rho}_{\mathrm{eq}} & =\boldsymbol{\rho}_{22} / \phi^{2} \\
\boldsymbol{\rho}_{s} & =\left(\boldsymbol{\rho}_{11}-\boldsymbol{\rho}_{12}^{2} \boldsymbol{\rho}_{22}^{-1}\right)+\gamma^{2} \boldsymbol{\rho}_{\mathrm{eq}}
\end{aligned}
$$

where $\mathbf{I}$ is the second order identity tensor, $R$ is the effective bulk modulus of the fluid phase and $Q$ indicates the coupling of the two phases volumic dilatation. Other definitions $^{35}$, more consistent with the 2007 formulation $^{5}$, can be found in Ref.34. For a complete anisotropic description the reader can refer to Hörlin et al. ${ }^{17}$.

Without loss of generality, all tensors are represented by matrices and expressed in their principal basis which reflect the transverse isotropy of the frame. The axis of the coordinate system: $\mathbf{e}_{x}$ is the isotropy axis and $\mathbf{e}_{y}$ and $\mathbf{e}_{z}$ define the plane of isotropy (see Fig. 1). Where the Biot effective densities $\boldsymbol{\rho}_{11}, \boldsymbol{\rho}_{12}, \boldsymbol{\rho}_{22}$ take the form of diagonal matrices

$$
\boldsymbol{\rho}_{i j}=\operatorname{diag} \rho_{i j}^{k}, \quad k=T, I, I,
$$

where $\rho_{i j}^{k}$ is given by

$$
\begin{aligned}
& \rho_{12}^{k}=\phi \rho_{f}\left(1-\alpha^{k}\right), \\
& \rho_{22}^{k}=\phi \rho_{f}-\rho_{12}^{k}, \\
& \rho_{11}^{k}=\rho_{1}-\rho_{12}^{k} .
\end{aligned}
$$

Here, $\rho_{1}=(1-\phi) \rho_{m}$ is the in vacuo bulk density whereas $\rho_{m}$ is the density of the matter constituting the solid phase and $\rho_{f}$ the density of the saturating fluid. It is assumed that these values are the same for all directions. The viscous losses are taken into account by coefficients $\alpha^{k}$ which are the dynamic tortuosity in the direction $k$, and these are given by the Johnson-Champoux-Allard model (this is reminded in the appendix A) and it is assumed they have the same TI as the frame $i e$ the same principal axis. An important consequence is that all second order tensors in the right hand side of Eq. (1) and in Eqs. (2)-(4) can commute since they have the same principal directions. 
For the skeleton, the in vacuo stress-strain relation for TI materials with Voigt notation, reads (see Chap. 10 in Allard et al. textbook ${ }^{2}$ ):

$$
\left(\begin{array}{l}
\hat{\sigma}_{x x} \\
\hat{\sigma}_{y y} \\
\hat{\sigma}_{z z} \\
\hat{\sigma}_{y z} \\
\hat{\sigma}_{x z} \\
\hat{\sigma}_{x y}
\end{array}\right)=\left(\begin{array}{cccccc}
C & F & F & 0 & 0 & 0 \\
F & B & A & 0 & 0 & 0 \\
F & A & B & 0 & 0 & 0 \\
0 & 0 & 0 & G_{I I} & 0 & 0 \\
0 & 0 & 0 & 0 & G_{T I} & 0 \\
0 & 0 & 0 & 0 & 0 & G_{T I}
\end{array}\right)\left(\begin{array}{c}
\varepsilon_{x x} \\
\varepsilon_{y y} \\
\varepsilon_{z z} \\
2 \varepsilon_{y z} \\
2 \varepsilon_{x z} \\
2 \varepsilon_{x y}
\end{array}\right)
$$

where $A, B, F, G_{T I}, G_{I I}$ and $C$, are the elasticity moduli. For the sake of completness, their explicit expressions are reminded in appendix B.

At this stage, some assumptions are suggested to simplify the governing equations. First, the type of materials considered here must have very small Poisson's ratios ${ }^{16,36}$ so that $A$ and $F$ terms can be neglected in the system (7), becoming diagonal. The remaining coefficients are then of the same order of magnitude as the solid elastic moduli, i.e. $C \sim E_{T}$ whereas $B \sim E_{I}$ and therefore $G_{I I} \sim 2 E_{I}$. It is also assumed that $G_{T I} \sim E_{T}$. Secondly, $E_{T}$ is considered much smaller than the bulk modulus of the fluid $K_{\text {eq }}{ }^{37}$. We can borrow from the limp frame model and neglect the stress field along the transverse direction. The solid and total displacements become linearly dependent as

$$
u_{T}=-\frac{\rho_{\mathrm{eq}}^{T}}{\rho_{s}^{T}} \gamma^{T} u_{T}^{w}
$$

Finally, we assume that $E_{I} \gg E_{T}$ : the limp model is no more suitable in the plane of isotropy and we consider the rigid frame equivalent fluid model in the $I$ directions. This hypothesis is to consider that the frame does not undergo any displacements, that reads

$$
\mathbf{u}_{I}=0
$$

With these assumptions, Eq. (1b) has as simpler form involving the total displacement only

$$
K_{\mathrm{eq}} \nabla\left(\nabla \cdot \mathbf{u}^{w}\right)=-\omega^{2} \boldsymbol{\rho} \mathbf{u}^{w}
$$

where the diagonal tensor

$$
\boldsymbol{\rho}=\operatorname{diag}\left(\rho_{\ell}^{T}, \rho_{\mathrm{eq}}^{I}, \rho_{\mathrm{eq}}^{I}\right)
$$

can be interpreted as a TI density tensor involving the limp density

$$
\rho_{\ell}^{T}=\rho_{\mathrm{eq}}^{T}\left(1-\frac{\rho_{\mathrm{eq}}^{T}\left(\gamma^{T}\right)^{2}}{\rho_{s}^{T}}\right),
$$

in the transverse direction and the classical rigid frame equivalent density in the plane of isotropy $\rho_{\mathrm{eq}}^{I}$. In the low frequency limit ${ }^{32}$, the rigid frame model density is dominated by its imaginary part and $\rho_{\text {eq }}^{I} \sim \mathrm{i} \sigma^{I} / \omega$ while the limp effective density tends to a real-valued constant equal to the total apparent mass of the equivalent fluid limp medium as $\rho_{\ell}^{T} \approx \rho_{1}+\phi \rho_{f}$ which can reach a high value depending on the frame added mass.
Recalling that the fluid pressure is obtained from the mass conservation equation ${ }^{18}$, i.e. $p=-K_{\mathrm{eq}} \nabla \cdot \mathbf{u}^{w}$, Eq. (10) can be recast into a scalar wave equation for the pressure

$$
\nabla \cdot(\tau \nabla p)+\frac{\omega^{2}}{K_{\mathrm{eq}}} p=0
$$

where we put $\boldsymbol{\tau}=\boldsymbol{\rho}^{-1}=\operatorname{diag}\left(\tau_{T}, \tau_{I}, \tau_{I}\right)$. A similar equation has been derived in the De Hoop's textbook ${ }^{25}$ (Chap. 6) and in the context of sonic crytals ${ }^{28}$ and metamaterials ${ }^{30}$. The approach has been extended to the description of pentamode materials involving nonscalar bulk moduli ${ }^{30,38,39}$. To the authors' knowledge, Eq. (13) which describes the wave propagation in transversely isotropic soft materials is a new result. The model, which relies on classical assumptions encountered both in rigid and limp frame models allows to capture the main characteristics of the wave motion in the plane of isotropy and along the transverse direction. It differs from other TI equivalent fluid models proposed in the literature $^{20-23}$ in that it allows to take into account the inertia of the frame in the soft direction.

Note that when the principal directions do not coincide with the coordinate system, the new density tensor is obtained via rotation. To illustrate this, let the $z$-axis be the axis of rotation and call $\Theta$ the angle between the $T$ direction and $\mathbf{e}_{x}$. In this new coordinate system,

$$
\boldsymbol{\tau}^{\prime}=\mathbf{R} \boldsymbol{\tau} \mathbf{R}^{\mathrm{T}}
$$

where

$$
\mathbf{R}=\left(\begin{array}{ccc}
\cos \Theta & \sin \Theta & 0 \\
-\sin \Theta & \cos \Theta & 0 \\
0 & 0 & 1
\end{array}\right)
$$

\section{B. Plane wave solutions in media of inifnite extent}

Plane waves are homogeneous solutions of Eq. (13) of the form $p=\mathrm{e}^{\mathrm{i} \mathbf{k} \cdot \mathbf{x}}$, where

$$
\mathbf{k}=k(\cos \theta, \sin \theta \cos \varphi, \sin \theta \sin \varphi),
$$

is the wavevector and $k=\omega / c$ is the wavenumber. After insertion into the wave equation, the dependence of the wave speed with the direction of propagation is given by

$$
c^{2}=K_{\mathrm{eq}}\left(\tau_{T} \cos ^{2} \theta+\tau_{I} \sin ^{2} \theta\right)
$$

which corresponds to an ellipsoid of revolution ${ }^{25}$ when effective densities are real-valued.

Real and imaginary parts of the slowness $1 / c$ are illustrated in Fig. 2 for three frequencies. Note only the first quadrant is considered for symmetry reasons. The material considered here is a glass wool and its acoustical and mechanical properties characteristics are given in Tables I and II (the conditions in which measurements have been made is discussed in Section III).

At low frequency, the attenuation, indicated by the slowness imaginary part, reaches a minimum along the $T$ 
direction and this was expected if we have in mind the asymptotic behaviour of the densities, as discusssed earlier. This is no longer the case at higher frequencies where the attenuation becomes smaller in the $I$ direction. This suggests that there should be a frequency where both attenuations, i.e. the imaginary part of the slowness, are equal. This scenario is well illustrated in Fig. 2(b) where attenuation is nearly contant regardless of the direction of propagation. Above a certain frequency, the high frequency behaviors of both models, i.e. limp and rigid frame, are nearly identical and the slight anisotropy stems from the material characteristics which differ depending on the stiff and soft directions.

\section{Guided waves}

In this section the case of a $2 \mathrm{D}$ rigid-walled waveguide of height $h$ filled with a TI fluid is investigated. We are looking for a guided mode propagating along the $\mathbf{e}_{x}$ direction (i.e. the waveguide axis), having the exponential form $p=\psi(y) \mathrm{e}^{\mathrm{i} \beta x}$ where

$$
\psi(y)=a \mathrm{e}^{\mathrm{i} \alpha_{1} y}+b \mathrm{e}^{\mathrm{i} \alpha_{2} y} .
$$

After insertion into the wave equation, transverse wavenumbers $\alpha_{1}$ and $\alpha_{2}$ must satisfy

$$
\tau_{y y} \alpha^{2}+2 \beta \tau_{x y} \alpha+\tau_{x x} \beta^{2}-\frac{\omega^{2}}{K_{\mathrm{eq}}}=0 .
$$

In order to take into account an angle $\Theta$ between the $T$ direction and the waveguide axis, tensor coefficients have the explicit form (see Eq. (14))

$$
\begin{aligned}
& \tau_{x x}=\tau_{T} \cos ^{2} \Theta+\tau_{I} \sin ^{2} \Theta, \\
& \tau_{y y}=\tau_{I} \cos ^{2} \Theta+\tau_{T} \sin ^{2} \Theta, \\
& \tau_{x y}=\sin \Theta \cos \Theta\left(\tau_{I}-\tau_{T}\right) .
\end{aligned}
$$

Applying rigid conditions at the wall, i.e. $\mathbf{u}^{w} \cdot \mathbf{e}_{y}=0$, leads to a boundary condition of mixed type

$$
\boldsymbol{\tau} \nabla p \cdot \mathbf{e}_{y}=\left(\mathrm{i} \beta \tau_{x y} \psi+\tau_{y y} \partial_{y} \psi\right) \mathrm{e}^{\mathrm{i} \beta x}=0, \quad y=0, h
$$

and non-trivial solutions exist if

$$
\sin \left(\frac{h \sqrt{\Delta}}{2 \tau_{y y}}\right)=0
$$

where

$$
\Delta=4 \tau_{y y} \frac{\omega^{2}}{K_{\mathrm{eq}}}+4 \beta^{2}\left(\tau_{x y}^{2}-\tau_{x x} \tau_{y y}\right)
$$

is the discriminant of the quadratic equation (19). Propagation constant for guided modes are then found explicitly as

$$
\beta_{n}= \pm \frac{\tau_{y y}}{\sqrt{\tau_{T} \tau_{I}}} \sqrt{\frac{\omega^{2}}{\tau_{y y} K_{\mathrm{eq}}}-\left(\frac{n \pi}{h}\right)^{2}} .
$$

The fact that right and left propagating modes are identical was expected for symmetry reasons. Note that the fundamental mode, $n=0$, is also function of the transverse coordinate $y$ except when $\Theta=0$ or $\pi / 2$. These two particular configurations are treated in Section IIIC.

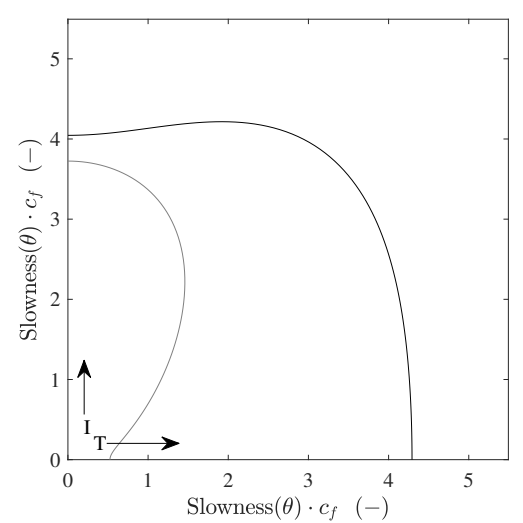

(a)

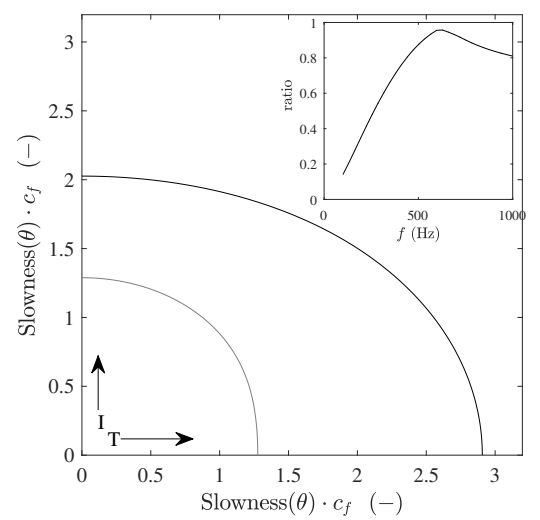

(b)

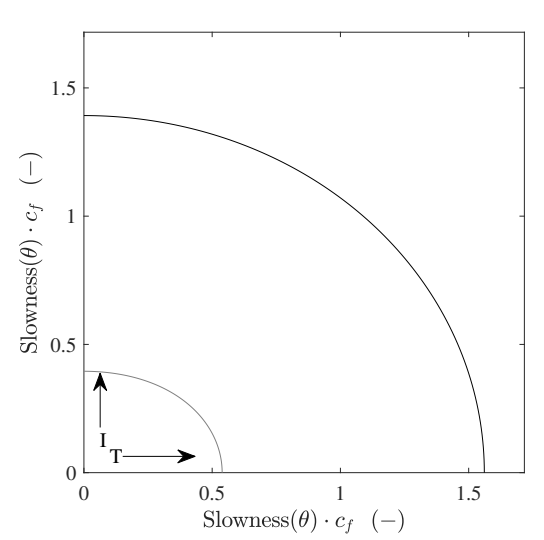

(c)

FIG. 2. Real — and imaginary part - of the slowness curves at a) $100 \mathrm{~Hz}$, b) $600 \mathrm{~Hz}$ and c) $5000 \mathrm{~Hz}$ normalized by the saturating fluid slowness $c_{f}^{-1}$. The inset in (b) shows the ratio between the min and the max of the slowness imaginary part with respect to the frequency.

\section{General case}

The wave propagation in the TI fluid can be solved numerically using the finite element method. The associated weak formulation, is obtained by multiplying the wave equation (13) by a test function $q$ and by integrating over the porous media occupying an arbitrary domain 
TABLE I. Acoustical parameters.

$$
\int_{\Omega} \nabla q \cdot(\boldsymbol{\tau} \nabla p) \mathrm{d} \Omega+\frac{\omega^{2}}{K_{\mathrm{eq}}} \int_{\Omega} q p \mathrm{~d} \Omega=\omega^{2} \int_{S} q \mathbf{u}^{w} \cdot \mathbf{n} \mathrm{d} S .
$$

For problems involving a surrounding fluid or another porous material modeled as an equivalent fluid, transmission conditions, i.e. continuity of pressure and total displacement, are automatically satisfied in the weak formulation so Eq. (27) remains valid over the whole computational domain and it suffices to modify the density tensor and bulk modulus accordingly.

In the present work, this formulation has been used for the examples described in sec. III.D. The FEM computations are carried out using Lagrange quadratic triangular finite elements in FreeFEM $++{ }^{40}$. Incident acoustic waves and non reflecting boundary conditions are prescribed using modal expansion ${ }^{41}$ for rigid ducts.

\section{EXPERIMENTAL VALIDATION}

In this section, acoustic and elastic parameters of the tested material are presented. Then, two configurations are investigated experimentally in order to assess the validity of the TI model. The first one concerns the absorption of an incident plane wave impinging on a fibrous slab placed at the end of a rigid duct, see Fig. 3(c). The second configuration shown in Fig. 3(b) corresponds to a splitter-type silencer in a rigid duct with a rectangular cross-section.

\section{A. Material characterization}

The tested material is a glass wool illustrated in Fig. 1. Each layer has a nominal thickness of $25.4 \mathrm{~mm}$ (1 inch). Experimental characterizations have been performed assuming that samples are extracted along the principal directions (see Fig. 1). In a more general case, note that suitable methods can be found in ${ }^{9,11,12}$. Even if elastic parameters are not required by the TI equivalent model, these will be useful later (in Sec. III.D) in order to evaluate the influence of elastic waves and establish the validity of the present scalar model.

The properties have been evaluated using several test benches at Roberval laboratory. The porosity is measured by using the pressure/mass method ${ }^{42}$. The resistivity is determined according to the method ISO 9053. The tortuosity is measured by ultrasonic method ${ }^{43}$ and the two characteristic lengths are obtained by indirect method based on the 3 microphones in-duct technique ${ }^{44}$. The parameters involved in the Johnson-ChampouxAllard model have been obtained for three samples. The mean values are reported in the Table I. Note that the standard deviation is lower than $12 \%$ which is in line with Ref.45.

The resistivity ratio between the $I$ and $T$ directions is found to be 0.44 which is close to theoretical prediction of $1 / 2$ given by Tarnow ${ }^{46}$ assuming that fibers can be modeled as parallel cylinders with equal radius. Note

\begin{tabular}{ccccccc}
\hline \hline $\begin{array}{c}\phi_{m} \\
-\end{array}$ & $\begin{array}{c}\sigma^{T} \\
{\left[\mathrm{Nm}^{-4} \mathrm{~s}\right]}\end{array}$ & $\begin{array}{c}\sigma^{I} \\
{\left[\mathrm{Nm}^{-4} \mathrm{~s}\right]}\end{array}$ & $\begin{array}{c}\alpha_{\infty} \\
-\end{array}$ & $\begin{array}{c}\Lambda^{T} \\
{[\mu \mathrm{m}]}\end{array}$ & $\begin{array}{c}\Lambda^{I} \\
{[\mu \mathrm{m}]}\end{array}$ & $\begin{array}{c}\Lambda^{\prime} \\
{[\mu \mathrm{m}]}\end{array}$ \\
\hline 0.98 & 38000 & 16720 & 1.0 & 29.5 & 50.1 & 70.8 \\
\hline \hline
\end{tabular}

TABLE II. Mechanical properties. Symbol "*” indicates that the corresponding value is extrapolated from Ref.16.

\begin{tabular}{ccccccc}
\hline \hline $\begin{array}{c}E_{T} \\
{[\mathrm{~Pa}]}\end{array}$ & $\begin{array}{c}E_{I} \\
{[\mathrm{~Pa}]}\end{array}$ & $\begin{array}{c}G_{T I} \\
{[\mathrm{~Pa}]}\end{array}$ & $\begin{array}{c}\nu_{I I} \\
-\end{array}$ & $\begin{array}{c}\nu_{T I} \\
-\end{array}$ & $\begin{array}{c}\eta \\
\rho_{1} \\
{\left[\mathrm{kgm}^{-3}\right]}\end{array}$ \\
\hline 670 & 142000 & $3520^{*}$ & 0 & 0 & 0.1 & 16 \\
\hline \hline
\end{tabular}

that when cylinders are oriented randomly in the plane of isotropy the ratio should be close to $2 / 3$. However Tarnow $^{47}$ measured a smaller ratio, about 0.5 , that he justifies by the inhomogeneous distribution of the fibers in space. The ratio between viscous characteristic lengths $\Lambda_{I} / \Lambda_{T}$ is 1.70 .

Poisson ratios are assumed to be zero as commonly admitted for fibrous materials ${ }^{16,36}$. Young's moduli are estimated by quasistatic method without static preload ${ }^{48}$. The mean values obtained for three samples can be found in Table II. The standard deviation for Young's modulus is around $8 \%$. The ratio $E_{T} / E_{I}$ is 211 , which is around 3 times the ratio found by Rice et al. ${ }^{16}$ so the anisotropy of our material is more pronounced. The value of the coefficient $G_{T I}$, which has not been measured, is assumed to be equal to $5.3 E_{T}$ as in Ref.16. This point is not crucial as it was checked, via numerical computation, that the value of $G_{T I}$ is not influential as long as it remains small in comparaison with $E_{I}$.

In order to anticipate the influence of skeleton elasticity, it is instructive to borrow from previous work ${ }^{37}$ and apply the Frame Stiffness Influence (FSI) criteria which indicates that limp model should apply when $\mathrm{FSI}_{\mathrm{r}}<0.1$. In the present case, it is found that $\mathrm{FSI}_{\mathrm{r}} \sim 0.003$ in the $I$ direction and $\mathrm{FSI}_{\mathrm{r}} \sim 0.7$ in the $T$ direction. Another parameter of particular interest to us is the phase decoupling frequency $f_{d}$ introduced in Ref.49 estimated as follows: $f_{d} \sim \frac{\max \left(\sigma^{k}\right) \phi^{2}}{2 \pi \rho_{1}} \sim 360 \mathrm{~Hz}$. This means that, the skeleton could be considered as motionless for fluid excitation above this frequency (if boundary conditions influence can be neglected). These considerations will be illustrated later in a practical configuration.

\section{B. Test bench and experimental procedure}

Measurements are made on a test bench (Fig. 3) designed for the acoustic multimodal characterization of test section in the presence (or not) of a low Mach number flow ${ }^{31,50}$. The duct facility is a rigid rectangular duct of $0.2 \mathrm{~m} \times 0.1 \mathrm{~m}$ section with an anechoic termination at each end. The results are given within the frequency band $[200 \mathrm{~Hz}-3.5 \mathrm{kHz}]$ which limits the number of propagative modes in the rigid duct to a maximum 

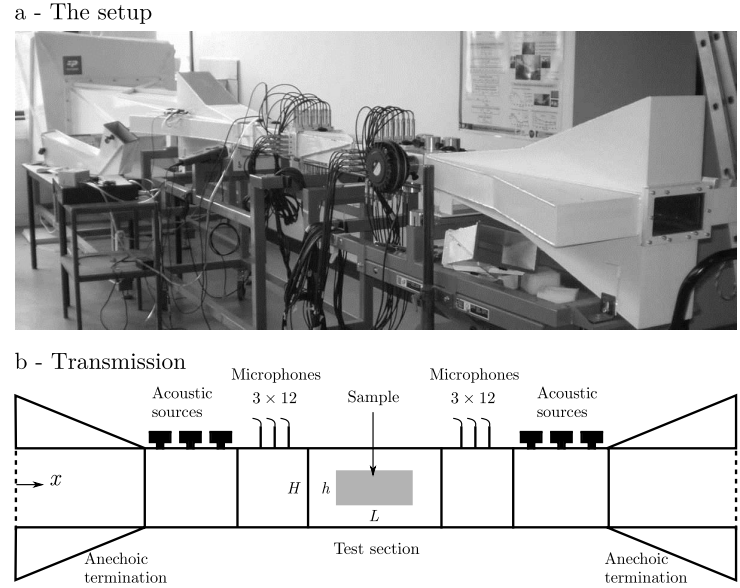

c - Absorption

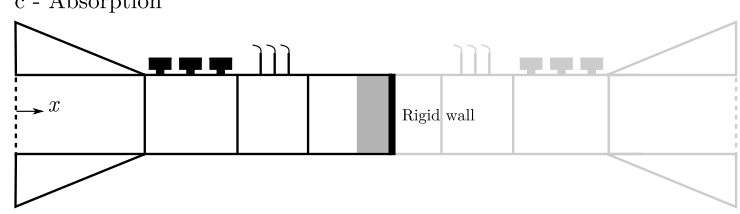

FIG. 3. Multimodal UTC test bench ${ }^{50}$ : (a) Experimental setup, (b) transmission configuration, (c) absorption configuration.

of $M_{s}=10$. The $2 M_{s}$-port scattering matrix, which contains the modal reflection and transmission coefficients, is measured using a multi-source method described in ${ }^{50}$. The set-up is generally used to study transmission problems, see Fig. 3(b). It can also be used for the measurement of absorbing coefficients, by sealing the duct at one end as shown in Fig. 3(c).

\section{Absorption at normal incidence}

A fibrous slab of rectangular shape and of length $L$ is inserted at the end of the rigid duct. Two orientations are investigated: $\Theta=0$ and $\Theta=\pi / 2$ with $L=5$ $\mathrm{cm}$ (made of two slightly prestressed $25.4 \mathrm{~mm}$ long fibrous layers) and $L=10 \mathrm{~cm}$ (made of four layers). Note that these configurations have been chosen so that surface impedance (assuming normal incidence) can be defined in the usual manner from Eq. (26). If the fibers are not aligned with the duct wall, guided waves in the slab depend on the transverse coordinate as in Eq. (18).

Comparison between analytical predictions of section II.C and experimental results are presented in Figs. 4(b) and 4(c). In each case, a good agreement is obtained, except near the cut-off frequencies of the duct, noticeably around $857 \mathrm{~Hz}$ and $1714 \mathrm{~Hz}$. In the first scenario $\Theta=0$, the $T$ direction is aligned with the duct axis and the fibrous slab acts as a limp material. Here, a sharp absorption peak can be observed around $220 \mathrm{~Hz}$ in Fig. 4(c) which illustrates resonance effects due to the rigid body motion of the skeleton. This corresponds to the quarter wavelength resonance frequency of the backed fibrous slab. When $\Theta=\pi / 2$, the $T$ direction is perpendicular to the duct axis and the slab acts as a rigid frame material. In this example, the anisotropy nature of the material has a strong impact on the absorbing properties and this could have been anticipated from the imaginary part of the slowness as discussed in section IIB. At higher frequencies, results are very similar, i.e. within $1 \mathrm{~dB}$, regardless of the fibers orientation as expected.
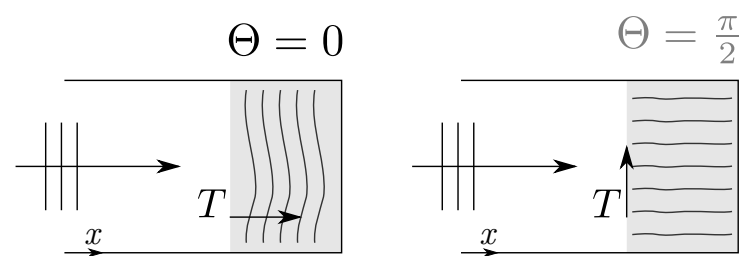

(a)

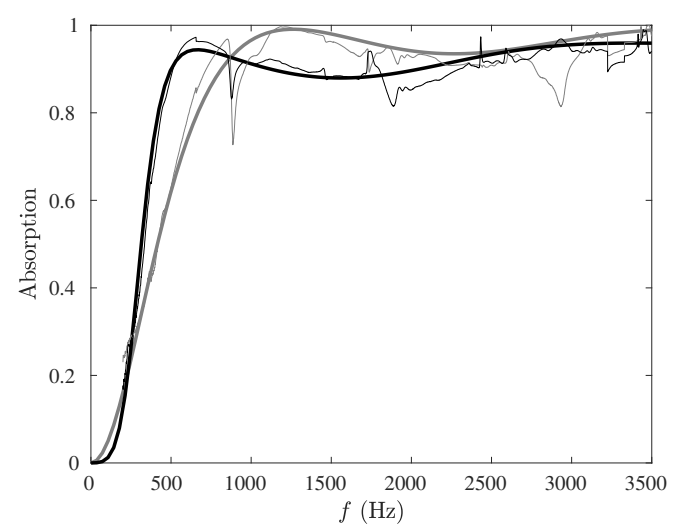

(b)

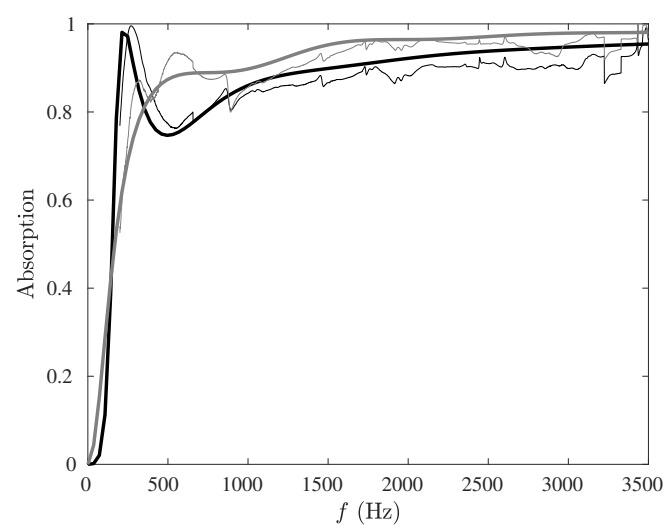

(c)

FIG. 4. Description of the configurations (a) and absorption coefficient measurements (thin line) and TI equivalent fluid model (thick line) for $\Theta=0$ and $\Theta=\frac{\pi}{2}$ and two sample thickness (b) $5 \mathrm{~cm}$, (c) $10 \mathrm{~cm}$.

\section{Silencer test case}

The silencer is made up of one splitter of length $L=30$ $\mathrm{cm}$ with thickness $h=10 \mathrm{~cm}$. The splitter is filled with glass wool and placed along the upper part of the inside wall of the duct, as shown in Fig. 5. Three orientations are tested and in all cases the porous layers are maintained by rigid fairings of size $100 \times 100 \mathrm{~mm}$. Acoustic 
performances of the silencer are assessed by measuring the Transmission Loss (TL) of an incident plane wave travelling from the left to the right end of the duct. Above the duct cut-off frequency, all propagating modes which are generated via mode conversion are taken into account in the calculation. For each configuration, two sets of measurements have been performed by assembling and deassembling the silencer in order to check the repeatability.

These measurements are compared to $2 \mathrm{D}$ finite element simulations based on the discretization of Eq. (27). For validation purposes, the full Transversely Isotropic Biot model using a pressure-displacement formulation proposed by Hörlin et al. ${ }^{17}$ is used ${ }^{51}$. The FEM computations are carried out using Lagrange quadratic triangular finite elements. Incident acoustic waves as well as non reflecting boundary conditions are prescribed similarly as in Sec. II.D. Note all computations are carried out with sliding boundary conditions at the wall and it was checked numerically that clamped boundary conditions do not have significant effects on the TL.

First, let us consider the direction $T$ orthogonal to the waveguide axis $(\Theta=\pi / 2)$ as depicted in Fig. 5(a). The sample is composed of 4 layers of $300 \times 100 \times 25.4 \mathrm{~mm}$ of wool. Results given in Fig. 6(a) show that the experiment has a good repeatability. This configuration is very well described by the TI equivalent fluid model and the TI Biot model. The very good agreement between both models reveals that the skeleton has no significant elastic contribution here. Note the high TL peak around the cut-off frequency which corresponds to a conversion effect among duct modes. All curves are in good agreement apart from a slight difference around $300 \mathrm{~Hz}$ where relatively high attenuation can be observed. This is related to a resonance effect along the $T$ direction in the wool. Here, the resonance quality factor is relatively high because, at low frequency, waves propagating along this direction are much less attenuated as discussed earlier.

The $T$ direction is now parallel to the waveguide axis $(\Theta=0)$ as depicted in Fig. 5(c). The sample is composed of 12 layers of $100 \times 100 \times 25.4 \mathrm{~mm}$. In this particular configuration, the presence of small air gaps between layers, skin effect (the fibrous material is usually denser near the surface) as well as slight variations in mechanical characteristics are unavoidable. Nevertheless, the experiment has a rather good repeatability. Both experimental curves show a bump around $400 \mathrm{~Hz}$. This behavior is not predicted by the TI equivalent fluid model simulation as opposed to the full TI Biot model which is in better agreement with experimental data. Thus this phenomenon, which appears around the decoupling frequency, is closely linked to a skeleton elastic resonance. This kind of resonances have already been predicted and studied in previous work ${ }^{41,52}$ related to cylindrical dissipative silencers.

Finally, we shall consider an oblique direction with $\Theta=$ $\pi / 4$ as depicted in Fig. 5(b). The sample is composed of 11 layers of different length and $25.4 \mathrm{~mm}$ thickness. The measurements are fairly repeatable and present a rather good agreement with both TI models though some elastic effects of the structure can be observed.
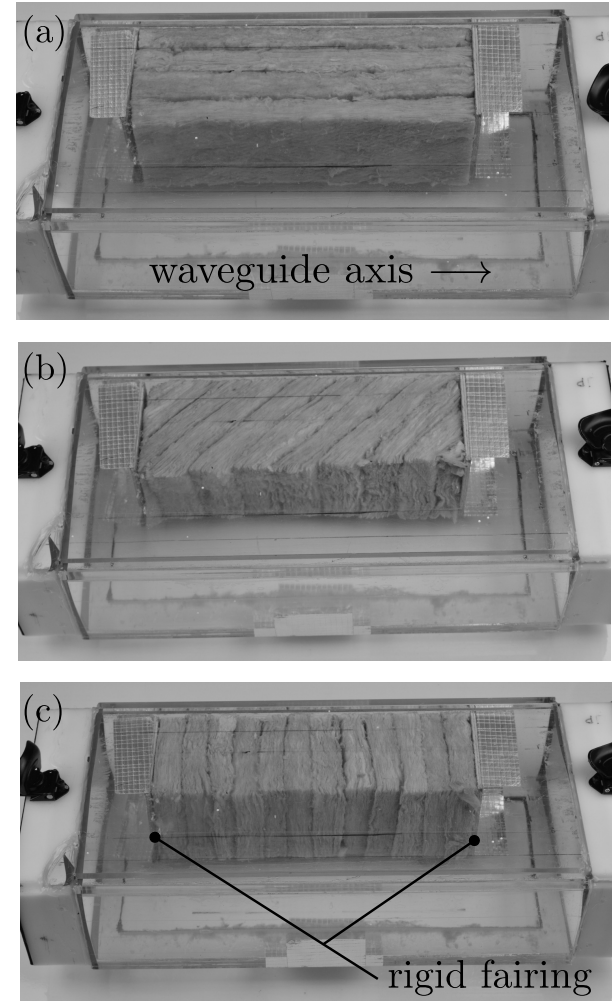

FIG. 5. Test section picture with the three orientations (a) $\Theta=\pi / 2$, (b) $\Theta=\pi / 4$, (c) $\Theta=0$.

Some of the results are conveniently reported in Fig. 7 which allow us to identify more clearly the effects of the fiber orientation on the TL. This shows that the equivalent fluid model proposed is sufficiently accurate to capture the essential behavior of dissipative silencers made with TI fibrous materials. As already explained, the occurrence of elastic resonances which can not be predicted by the model originates from the motion of the solid frame. If in principle, the full Biot theory could be employed to observe these phenomena, their effect on the TL remain moderate. Furthermore, they are difficult to predict accurately, both in frequency and amplitude as they must rely on the knowledge of elastic parameters as well as some inherent uncertainties due to the mounting conditions and some material inhomogeneities.

Above the cut-off frequency, the predicted TL for the three orientations are in good agreement with experimental data with a maximum discrepancy of about $1 \mathrm{~dB}$.

It may be noticed that all TL curves intersect around $400 \mathrm{~Hz}$ which suggests that the wave attenuation is nearly isotropic at this frequency. This is somewhat in line with results of Fig. 2, showing that isotropy of the attenuation is reached around $600 \mathrm{~Hz}$ in the porous material.

\section{CONCLUSION}

In this paper a new equivalent fluid model is proposed in order to take into account the transverse isotropic behavior of some porous materials like fibrous wool which 


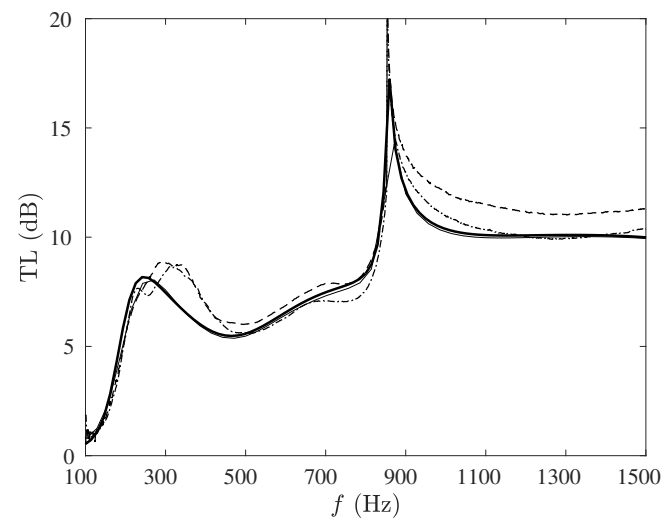

(a)

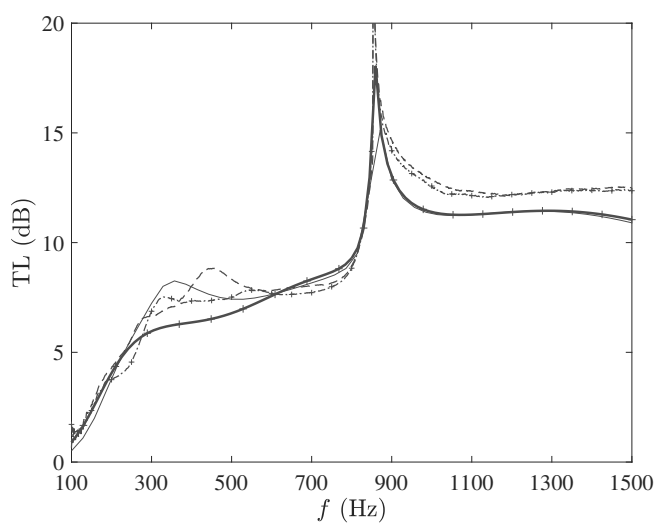

(b)

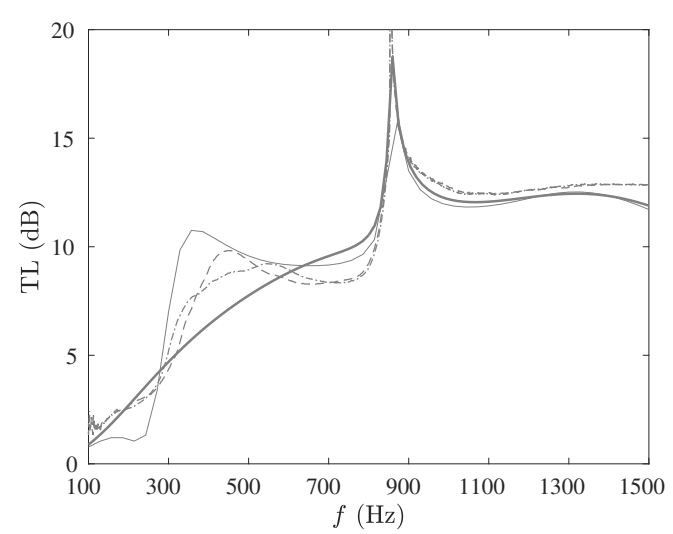

(c)

FIG. 6. Transmission loss for the three tested orientations. (a) $\Theta=\pi / 2$, (b) $\Theta=\pi / 4$, (c) $\Theta=0$,. - - experiment \#1, - - experiment \#2, - TI equivalent fluid FEM model and — Biot TI FEM model.

present a strong contrast between the plane of isotropy parallel to the fibers and the transverse direction. It leads to a scalar wave equation for the pressure involving effective densities which takes the form of a tensor and permits to combine both limp and rigid frame behaviors of the material. Experimental validations are carried out for two configurations. The first one concerns the absorption of an incident plane wave impinging on a fibrous slab.

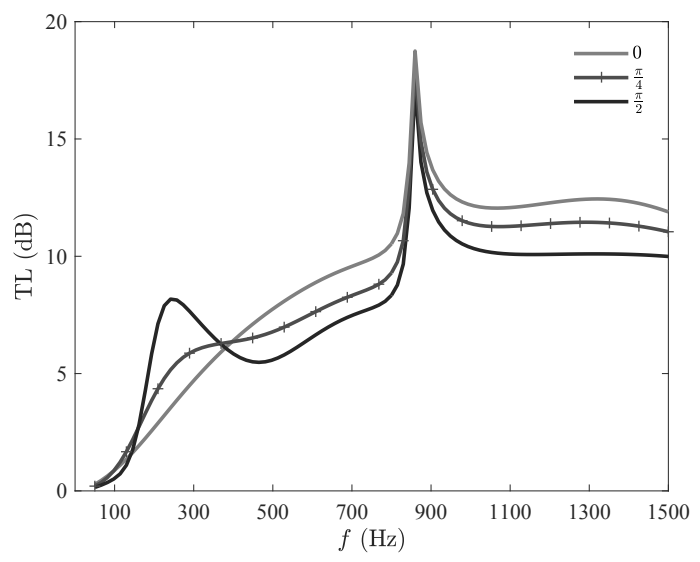

(a)

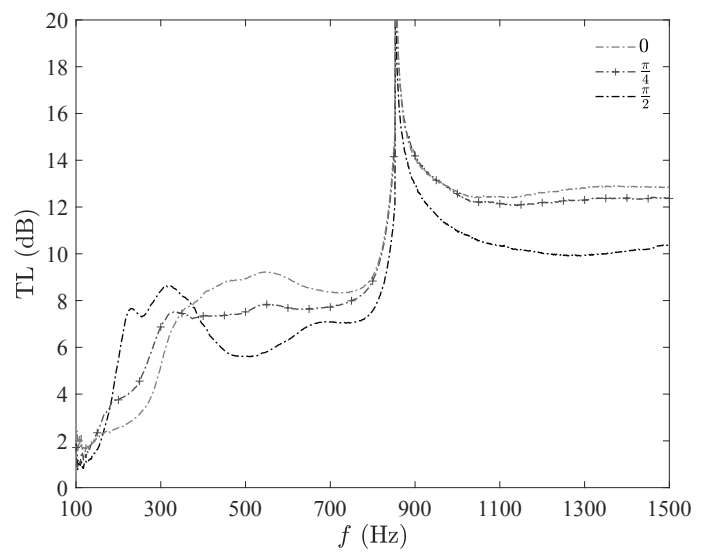

(b)

FIG. 7. Comparison between three configurations for (a)TI equivalent fluid model, (b) experimental data \#2.

The second configuration corresponds to the Transmission Loss (TL) of a splitter-type silencer in a rigid duct with a rectangular cross-section. In both cases, we show that the material anisotropy has a significant impact on the sound absorbing properties as function of orientation. The equivalent fluid model permits the analysis of silencers made with a certain class of TI fibrous materials, having arbitrarily specified orientation of fibers, without the knowledge of its elastic parameters. A comparison with the full TI Biot's model revealed that some elastic resonances might appear but their effects remain moderate on the TL. They are also hard to predict accurately as they depend on numerous uncertain parameters such as the mounting conditions, material inhomogeities and elastic parameters.

For other kind of TI materials, a similar approach could be followed in order to derive a transverse isotropic equivalent fluid model combining limp (resp. rigid frame) model behaviors with different densities, in all directions.

In view of some preliminary results shown in this work or in Refs.53, 54, TI absorbing materials should be studied further in order to increase sound attenuation in large dissipative splitter silencers used in industrial applica- 
tions and work is ongoing by the authors to exploit this and seek for tailored made solutions.

\section{Acknowledgments}

This work was partly funded by the ANR Project METAUDIBLE No. ANR-13-BS09-0003-01 funded jointly by ANR and FRAE.

\section{APPENDIX A: JOHNSON-CHAMPOUX-ALLARD MODEL}

Porous materials with a rigid skeleton such as the porous material involved in this paper, are well described by the Johnson-Champoux-Allard ${ }^{2}$ (Chap. 5) equivalent fluid model. The equivalent bulk modulus of the TI fluid, is not affected by the anisotropy ${ }^{10}$ and reads

$$
K_{\mathrm{eq}}=\frac{\gamma P_{0} / \phi}{\gamma-(\gamma-1)\left[1+\mathrm{i} \frac{8 \eta}{\Lambda^{\prime 2} \operatorname{Pr} \omega \rho_{f}}\left(1-\mathrm{i} \rho_{f} \frac{\omega \operatorname{Pr} \Lambda^{\prime 2}}{16 \eta}\right)^{1 / 2}\right]^{-1}} .
$$

Where $\gamma$ is the air specific heat ratio and $P_{0}$ is the atmospheric pressure, $\operatorname{Pr}$ is the Prandtl number, $\Lambda^{\prime}$ is the thermal length and $\eta$ is the dynamic viscosity.

The viscous dissipation effects depend of the air flow resistivity $\boldsymbol{\sigma}$ and of the viscous characteristic length $\boldsymbol{\Lambda}$. It has been measured and shown theoretically ${ }^{12-14,46,47}$ that the flow resistivity is larger in the stacking direction than in the in-plane direction. It is classical to express the air flow resistivity as a second-order tensor which is diagonal in the orthogonal basis of principal directions:

$$
\boldsymbol{\sigma}=\operatorname{diag} \sigma^{k}, \quad k=T, I, I
$$

and similarly

$$
\mathbf{\Lambda}=\operatorname{diag} \Lambda^{k}, \quad k=T, I, I .
$$

In the high frequency limit, the tortuosity is considered as identical in all directions and $\alpha_{\infty}^{k}=\alpha_{\infty}=1$.

All viscous effects can be included in the equivalent dynamic tortuosity $\left(\mathrm{e}^{-\mathrm{i} \omega t}\right)$ in the principal direction $k$

$$
\alpha^{k}=\alpha_{\infty}^{k}\left(1+\frac{\mathrm{i} \phi \sigma^{k}}{\alpha_{\infty}^{k} \rho_{f} \omega} G_{J}^{k}(\omega)\right)
$$

Here, $G_{J}^{k}(\omega)=\sqrt{1-\frac{4 \mathrm{i}\left(\alpha_{\infty}^{k}\right)^{2} \eta \rho_{f} \omega}{\left(\sigma^{k} \Lambda^{k} \phi\right)^{2}}}, \phi$ is the porosity, $\sigma^{k}$ is the flow resistivity, $\Lambda^{k}$ is the viscous length in the direction $k$.

\section{APPENDIX B: DEFINITION OF THE ELASTIC MODULUS}

For linear transverse isotropy, the elasticity stiffness matrix has five independent constants. Here we follow ${ }^{2,55}$ (Chap. 10) and we use the Young's Modulus in the isotropy plane $E_{I}$, the Young's modulus in the normal direction $E_{T}$, the shear modulus normal to the plane of isotropy $G_{T I}$. Note that the shear modulus for the plane of isotropy $G_{I I}$ is not an independent coefficient unlike $G_{T I}$. The Poisson's ratio $\nu_{I I}$ characterizes the transverse strain in the plane of isotropy for a tensile stress in the same plane. Finally $\nu_{T I}$ is the Poisson's ratio corresponding to the transverse strain in the plane of isotropy due to a tensile stress normal to it. The coefficient of the rigidity matrix given in Eq. (7) reads

$$
\begin{aligned}
A & =\frac{E_{I}\left(E_{T} \nu_{I I}+E_{I} \nu_{T I}^{2}\right)}{\left(1+\nu_{I I}\right)\left(E_{T}-E_{T} \nu_{I I}-2 E_{I} \nu_{T I}^{2}\right)}, \\
F & =\frac{E_{I} E_{T} \nu_{T I}}{E_{T}-E_{T} \nu_{I I}-2 E_{I} \nu_{T I}^{2}}, \\
C & =\frac{E_{T}^{2}\left(1-\nu_{I I}\right)}{E_{T}-E_{T} \nu_{I I}-2 E_{I} \nu_{T I}^{2}}, \\
G_{I I} & =\frac{E_{I}}{2\left(1+\nu_{I I}\right)}, \\
B & =2 G_{I I}+A .
\end{aligned}
$$

1 M. A. Biot, "Mechanics of deformation and acoustic propagation in porous media," J. Appl. Phys. 33(4), 1482-1498 (1962).

2 J.-F. Allard and N. Atalla, Propagation of Sound in Porous Media: Modeling Sound Absorbing Materials (second edition) (John Wiley \& Sons, Chichester, 2009), p. 372.

3 L. L. Beranek, "Acoustical properties of homogeneous, isotropic rigid tiles and flexible blankets," J. Acoust. Soc. Am. 19(4), 556-568 (1947).

4 O. Doutres, N. Dauchez, J.-M. Génevaux, and O. Dazel, "Validity of the limp model for porous materials: a criterion based on the Biot theory," J. Acoust. Soc. Am. 122(4), 2038-2048 (2007).

5 O. Dazel, B. Brouard, C. Depollier, and S. Griffiths, "An alternative Biot's displacement formulation for porous materials," J. Acoust. Soc. Am. 121(6), 3509-3516 (2007) doi: $\backslash$ nolinkurl\{10.1121/1.2734482\}.

6 R. Panneton, "Comments on the limp frame equivalent fluid model for porous media," J. Acoust. Soc. Am. 122(6), EL217-EL222 (2007).

7 A. Geslain, J. P. Groby, O. Dazel, S. Mahasaranon, K. V. Horoshenkov, and A. Khan, "An application of the Peano series expansion to predict sound propagation in materials with continuous pore stratification," J. Acoust. Soc. Am. 132(1), 208-215 (2012).

8 G. Gautier, L. Kelders, J. P. Groby, O. Dazel, L. De Ryck, and P. Leclaire, "Propagation of acoustic waves in a onedimensional macroscopically inhomogeneous poroelastic material," J. Acoust. Soc. Am. 130(3), 1390-1398 (2011).

9 C. Van der Kelen, J. Cuenca, and P. Göransson, "A method for characterisation of the static elastic properties of the porous frame of orthotropic open-cell foams," Inter. J. Engin. Sci. 86(0), 44 - 59 (2015) doi: \nolinkurl\{10. 1016/j.ijengsci.2014.10.005\}.

10 M. Melon, E. Mariez, C. Ayrault, and S. Sahraoui, "Acoustical and mechanical characterization of anisotropic opencell foams," J. Acoust. Soc. Am. 104(5), 2622-2627 (1998) doi: \nolinkurl\{10.1121/1.423897\}.

11 J. Cuenca, C. Van der Kelen, and P. Göransson, "A general methodology for inverse estimation of the elastic and anelastic properties of anisotropic open-cell porous materials - with application to a melamine foam," J. Appl. Phys. 
115(8), - (2014) doi: \nolinkurl\{10.1063/1.4865789\}.

12 C. Van der Kelen and P. Göransson, "Identification of the full anisotropic flow resistivity tensor for multiple glass wool and melamine foam samples," J. Acoust. Soc. Am. 134(6), 4659-4669 (2013) doi: \nolinkurl\{10.1121/1. $4824841\}$.

13 H. Tuan Luu, C. Perrot, and R. Panneton, "Influence of porosity, fiber radius and fiber orientation on the transport and acoustic properties of random fiber structures," Acta Acust. United Ac. 103(6), 1050-1063 (2017).

${ }^{14}$ H. Tuan Luu, C. Perrot, V. Monchiet, and R. Panneton, "Three-dimensional reconstruction of a random fibrous medium: Geometry, transport, and sound absorbing properties," J. Acoust. Soc. Am. 141(6), 4768-4780 (2017) doi: \nolinkurl\{10.1121/1.4989373\}.

15 B. P. Semeniuk and P. Göransson, "Microstructure based estimation of the dynamic drag impedance of lightweight fibrous materials," J. Acoust. Soc. Am. 141(3), 1360-1370 (2017) doi: \nolinkurl\{10.1121/1.4976814\}.

${ }^{16}$ H. Rice and P. Göransson, "A dynamical model of light fibrous materials," Int. J. Mech. Sci. 41(4), 561 - 579 (1999) doi: \nolinkurl\{10.1016/S0020-7403(98)00082-4\}.

17 N.-E. Hörlin and P. Göransson, "Weak, anisotropic symmetric formulations of Biot's equations for vibro-acoustic modelling of porous elastic materials," Int. J. Num. Meth. Eng. 84(12), 1519-1540 (2010) doi: \nolinkurl\{10.1002/ nme. 2955\}.

18 P. Khurana, L. Boeckx, W. Lauriks, P. Leclaire, O. Dazel, and J. F. Allard, "A description of transversely isotropic sound absorbing porous materials by transfer matrices," J. Acoust. Soc. Am. 125(2), 915-921 (2009) doi: \nolinkurl\{10.1121/1.3035840\}.

19 J. P. Parra Martinez, O. Dazel, P. Göransson, and J. Cuenca, "Acoustic analysis of anisotropic poroelastic multilayered systems," J. Appl. Phys. 119(8), 084907 (2016) doi: \nolinkurl\{10.1063/1.4942443\}.

20 J. Pyett, "The acoustic impedance of a porous layer at oblique incidence," Acustica 3(6), 375-382 (1953).

${ }^{21} \mathrm{~K}$. Attenborough, "The prediction of oblique-incidence behaviour of fibrous absorbents," J. Sound Vib. 14(2), 183 - 191 (1971) doi: \nolinkurl\{10.1016/0022-460X(71) 90383-X\}.

22 J. Allard, R. Bourdier, and A. L'Esperance, "Anisotropy effect in glass wool on normal impedance in oblique incidence," J. Sound Vib. 114(2), 233 - 238 (1987) doi: \nolinkurl\{10.1016/S0022-460X (87)80150-5\}.

23 J. Tran-Van, "Etude de l'influence de l'isotropie transverse dans les laines minérales sur la transmission acoustique des parois multicouches (influence of transverse isotropy in mineral wool on acoustic transmission through multilayer panels)," Ph.D. thesis, Université de Poitiers, 2004.

24 M. Schoenberg and P. N. Sen, "Properties of a periodically stratified acoustic half-space and its relation to a Biot fluid," J. Acoust. Soc. Am. 73(1), 61-67 (1983).

25 A. T. De Hoop, Handbook of Radiation and Scattering of Waves (second edition) (Academic Press, London, 1995), p. 1083.

26 A. J. Leggett, "A theoretical description of the new phases of liquid ${ }^{3} \mathrm{He}$," Rev. Mod. Phys. 47, 331-414 (1975) doi: \nolinkurl\{10.1103/RevModPhys.47.331\}.

27 D. L. Johnson, "Equivalence between fourth sound in liquid He II at low temperatures and the Biot slow wave in consolidated porous media," Appl. Phys. Lett. 37(12), 1065-1067 (1980) doi: \nolinkurl\{10.1063/1.91878\}.

28 D. Torrent and J. Sánchez-Dehesa, "Anisotropic mass density by two-dimensional acoustic metamaterials," New J.
Phys. 10(2), 023004 (2008) doi: \nolinkurl\{10.1088/ 1367-2630/10/2/023004\}.

29 D. Torrent and J. Sánchez-Dehesa, "Sound scattering by anisotropic metafluids based on two-dimensional sonic crystals," Phys. Rev. B 79, 174104 (2009) doi: \nolinkurl\{10.1103/PhysRevB.79.174104\}.

30 A. N. Norris, "Acoustic metafluids," J. Acoust. Soc. Am. 125(2), 839-849 (2009) doi: \nolinkurl\{10.1121/ $1.3050288\}$.

31 R. Binois, E. Perrey-Debain, N. Dauchez, B. Nennig, J.-M. Ville, and G. Beillard, "On the efficiency of parallel baffletype silencers in rectangular ducts: prediction and measurement," Acta Acust. United Ac. 101, 520-530 (2015) doi: \nolinkurl\{10.3813/AAA.918849\}.

32 B. Nennig, R. Binois, E. Perrey-Debain, and N. Dauchez, "A homogenization method used to predict the performance of silencers containing parallel splitters," J. Acoust. Soc. Am. 137(6), 3221-3231 (2015).

33 M. A. Biot, "Theory of propagation of elastic waves in a fluid-saturated porous solid. I. low-frequency range. II. higher frequency range," J. Acoust. Soc. Am. 28(2), 168191 (1956).

34 O. Dazel, F.-X. Bécot, and L. Jaouen, "Biot effects for sound absorbing double porosity materials," Acta Acust. United Ac. 98(4), 567-576 (2012).

35 Note there is a typo in Eq. (1e) and (1f).

${ }^{36} \mathrm{~V}$. Tarnow, "Dynamic measurements of the elastic constants of glass wool," J. Acoust. Soc. Am. 118(6), 36723678 (2005) doi: \nolinkurl\{10.1121/1.2118267\}.

37 O. Doutres, N. Dauchez, J.-M. Génevaux, and O. Dazel, "A frequency independent criterion for describing sound absorbing materials by a limp frame model," Acta Acust. United Ac. 95(4), 178-181 (2009) doi: \nolinkurl\{10. 3813/AAA. 918139\}.

${ }^{38}$ G. W. Milton and A. V. Cherkaev, "Which elasticity tensors are realizable?," J. Eng. Mater. Technol. 117(4), 483493 (1995) doi: \nolinkurl\{10.1115/1.2804743\}.

39 M. Kadic, T. Bückmann, N. Stenger, M. Thiel, and M. Wegener, "On the practicability of pentamode mechanical metamaterials," Appl. Phys. Lett. 100(19), 191901 (2012).

40 F. Hecht, "New development in FreeFem++," J. Numer. Math. 20(3-4), 251-265 (2012).

41 B. Nennig, M. Ben Tahar, and E. Perrey-Debain, "A displacement-pressure finite element formulation for analyzing the sound transmission in ducted shear flows with finite poroelastic lining," J. Acoust. Soc. Am. 130(1), 42$51(2011)$.

42 Y. Salissou and R. Panneton, "Pressure/mass method to measure open porosity of porous solids," Journal of applied physics 101(12), 124913 (2007).

43 A. Moussatov, C. Ayrault, and B. Castagnède, "Porous material characterization-ultrasonic method for estimation of tortuosity and characteristic length using a barometric chamber," Ultrasonics 39(3), 195-202 (2001).

44 O. Doutres, Y. Salissou, N. Atalla, and R. Panneton, "Evaluation of the acoustic and non-acoustic properties of sound absorbing materials using a three-microphone impedance tube," Applied Acoustics 71(6), 506-509 (2010).

45 F. Pompoli, P. Bonfiglio, K. V. Horoshenkov, A. Khan, L. Jaouen, F.-X. Bécot, F. Sgard, F. Asdrubali, F. D'Alessandro, J. Hübelt, N. Atalla, C. K. Amédin, W. Lauriks, and L. Boeckx, "How reproducible is the acoustical characterization of porous media?," J. Acoust. Soc. Am. 141(2), 945-955 (2017) doi: \nolinkurl\{10. $1121 / 1.4976087\}$. 
46 V. Tarnow, "Airflow resistivity of models of fibrous acoustic materials," J. Acoust. Soc. Am. 100(6), 3706-3713 (1996) doi: \nolinkurl\{10.1121/1.1476686\}.

47 V. Tarnow, "Measured anisotropic air flow resistivity and sound attenuation of glass wool," J. Acoust. Soc. Am. 111(6), 2735-2739 (2002) doi: \nolinkurl\{10.1121/1. $1476686\}$.

${ }^{48}$ N. Dauchez, M. Etchessahar, and S. Sahraoui, "On measurement of mechanical properties of sound absorbing materials," in 2nd Biot Conference on Poromechanics, Grenoble, France (2002), pp. 1-4.

49 C. Zwikker and C. W. Kosten, Sound Absorbing Materials (Elsevier, New York, 1949), p. 174.

50 H. Trabelsi, N. Zerbib, J.-M. Ville, and F. Foucart, "Passive and active acoustic properties of a diaphragm at low mach number," Eur. J. Comp. Mech. 20(1-4), 49-71 (2011).

51 In the present implementation, it is assumed that the dilatation coupling coefficient $Q$ is a scalar quantity. This assumption is valid when the frame material bulk modulus is very stiff.

52 B. Nennig, E. Perrey-Debain, and M. Ben Tahar, "A mode matching method for modelling dissipative silencers lined with poroelastic materials and containing mean flow," J. Acoust. Soc. Am. 128(6), 3308-3320 (2010).

53 T. Bravo and C. Maury, "Sound attenuation and absorption by anisotropic fibrous materials: Theoretical and experimental study," J. Sound Vib. 417, 165 - 181 (2018) doi: \nolinkurl\{10.1016/j.jsv.2017.11.037\}.

54 A. Nayfeh and J. Sun, "Sound attenuation in twodimensional ducts with anisotropic liners," J. Sound Vib. 41(4), 421 - 432 (1975) doi: \nolinkurl\{10.1016/ S0022-460X (75) 80106-4\}.

55 A.-D. Cheng, "Material coefficients of anisotropic poroelasticity," Int. J. Rock Mech. Min. Sci. 34(2), 199 - 205 (1997) doi: \nolinkurl\{10.1016/S0148-9062(96)00055-1\}. 